\title{
Sintering Time Effect on the Electrical Properties of Barium Titanium Ferrite
}

\author{
A.M. El-Rifae \\ Physics Department, Faculty of Girls for Arts, Science and Education, \\ Ain Shams Univ., Cairo, Egypt.
}

Polycrystalline ferrite $\mathrm{Ba}_{x} \mathrm{Ti}_{4-2 x} \mathrm{Fe}_{2 x} \mathrm{O}_{8}$ (x= 0.1, 0.3 and 0.5) with hollandite structure was synthesized by the standard double sintering ceramic technique. The changes of sintering time are taken into consideration showing its effect on the behavior of the sample. The electrical properties (real \& imaginary parts of dielectric constant, and ac. resistivity) were studied at different temperature as a function of frequencies. The calculated charge carrier mobility indicated that the behavior of the sample is similar to that of semiconductors. The variation of the electric dipole moment with sintering time is observed to be related to $\varepsilon$. The Seebeck voltage coefficient was studied to confirm the type of charge carriers that participate in the conduction processes. 


\section{Introduction}

Mixed ferrites of hollandite structure are the object of many investigations due to their interesting physical properties and technical use. In the past few years, the crystal structure of barium-titanium ferrite $\mathrm{Ba}_{\mathrm{x}} \mathrm{Ti}_{4}$ ${ }_{2 x} \mathrm{Fe}_{2 \mathrm{x}} \mathrm{O}_{8}$ with $\mathrm{x}=0.65$ was determined [1,2]. These compounds have structure characterized by rectangular tunnels, which is rutile structure affording open tunnel formed by two edge sharing metal canted octahedra. Y.Abbas et al. [3] studied the Mossbauer, electric and magnetic properties of Ba-Ti ferrite. H.Casimir [4] found some information about Ba-ferrite on how the saturation magnetization is affected by the substitution of other ions in metal (M) structure. Also, it was found that both the Curie point $\left(450^{\circ} \mathrm{C}\right)$ and the saturation magnetization of low temperature rapidly decrease with increasing the ordered parameters $(\delta)$. The resultant magnetic moment per formula unit of $\mathrm{BaFe}_{12} \mathrm{O}_{19}$ is equal to the sum of the moments of the seven octahedral ions and the ion in the layer containing $\mathrm{Ba}$, reduced by the moments of two octahedral and two tetrahedral ions which are oppositely oriented to them [5].

The lattice parameters were found to be $a=10.205^{\circ} \mathrm{A}, \mathrm{b}=2.975^{\circ} \mathrm{A}$ and $c=9.940^{\circ} \mathrm{A}$ and $\beta=90.85^{\circ}$ and the lattice contains two formula per unit cell. The $\mathrm{Ti}$ and $\mathrm{Fe}$ atoms are randomly distributed over one crystallographic site and are octahedrally coordinated by oxygen atoms. Barium titanium ferrite as a hollandite structure is often slightly deformed to monoclinic symmetry [1,2]. The structure is described as $\mathrm{O}_{2 \mathrm{~h}}^{3}-\mathrm{I}_{2} / \mathrm{m}$ with barium ions in $2(\mathrm{~b})$ and the titanium ions and the oxygen ions in 4(i) [6], because of the small deviation from tetragonal symmetry.

The electrical properties of ferrites depend on many parameters including the sintering temperature, chemical composition, microstructure and the preparation conditions. In the present work, one tries to find a correlation between the electrical properties and sintering time.

\section{Experimental}

The ferrites $\mathrm{Ba}_{\mathbf{x}} \mathrm{Ti}_{4-2 \mathrm{x}} \mathrm{Fe}_{2 \mathrm{x}} \mathrm{O}_{\mathbf{8}}$ (where $\mathrm{x}=0.1,0.3$, and 0.5) were prepared by using the double sintering standard ceramic technique [7]. Analytical reagent grade $\mathrm{BaCo} 3, \mathrm{Fe}_{2} \mathrm{O}_{3}$ and $\mathrm{TiO}_{2}$ were taken as starting materials.

Stoichiometric ratios of these materials were grounded in an agate mortar for 2 hours and transferred to an electric ball mill for 4 hours and then heated at $850^{\circ} \mathrm{C}$ for five hours. The mixture was grounded again to a very fine powder by using an agate mortar for about two hours, transferred to an electric 
shaker for another two hours, pressed into pellets of diameter $10 \mathrm{~mm}$ and thickness $1.8 \mathrm{~mm}$ under a pressure of $15 \times 10^{11} \mathrm{~N} / \mathrm{m}^{2}$. After that, the pellets were sintered in a Lenton furnace UAF $16 / 5$ (England) with microprocessor 818P in air at $1230^{\circ} \mathrm{C}$ at a heating rate of $6^{\circ} \mathrm{C} / \mathrm{min}$ for different sintering times of $(8 \mathrm{~h}$, $20 \mathrm{~h}$, and $33 \mathrm{~h}$ ) and then cooled to room temperature at the same rate of heating.

The real and imaginary parts of the dielectric constant as well as the ac. electrical resistivity measurements were carried out using the LCR bridge, type HIOKI 3530 (Japan). The two surfaces of each pellet were polished, coated with silver paste and checked for good conduction.

The temperature of the samples was measured using K-type thermocouple connected to digital thermometer (type Digi-sense U.S.A) with accuracy better than $\pm 1^{\circ} \mathrm{C}$.

\section{Results and discussion:}

Figure (1) is a typical curve which correlates the variation of the real part of dielectric constant $\varepsilon^{\prime}$ to temperature for the mixed ferrite $\mathrm{Ba}_{\mathrm{x}} \mathrm{Ti}_{4-2 \mathrm{x}} \mathrm{Fe}_{2 \mathrm{x}} \mathrm{O}_{8}$ (where $\mathrm{x}=0.5$ ) at sintering time of 20 hours as a function of frequency $(10 \mathrm{kHz}-$ -- $900 \mathrm{kHz}$ ). The figure shows that the values of $\varepsilon^{\prime}$ are nearly constant with temperature up to $\cong 380 \mathrm{~K}$ for all frequencies since the thermal response of the induced dipoles is negligible.

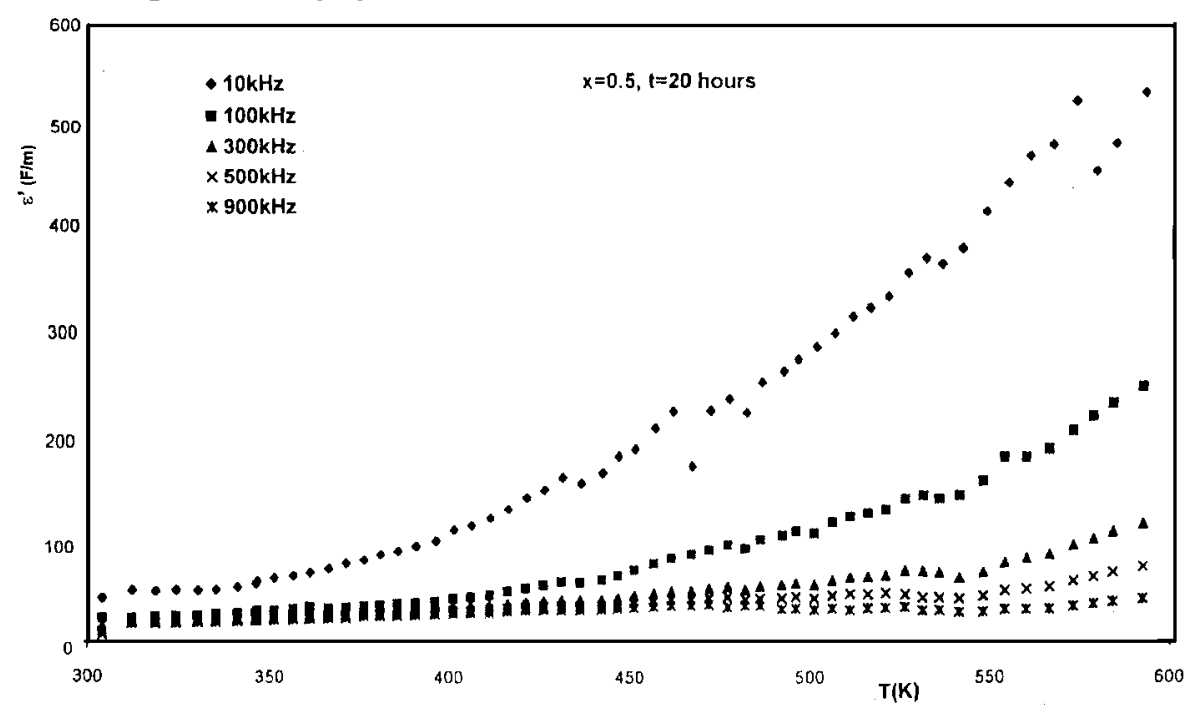

Fig. (1) : The real part of the dielectric constant $\varepsilon^{\prime}$ vs. the absolute temperature for concentration $\mathrm{x}=0.5$ and sintering time $\mathrm{t}=20$ hour as a function of frequency. 
Above $380 \mathrm{~K}$ the thermal energy becomes sufficient enough to help the valence variation of metal ions forming more dipoles with the anions. These dipoles are aligned in the field direction. In this case, the polarizability of the system was increased and the response appears in the form of increasing $\varepsilon^{\prime}$ with temperature. From the figure, it is also clear that increasing the applied frequency will increase the variation of the field accompanied with it and the electron exchange between $\mathrm{Fe}^{2+}$ and $\mathrm{Fe}^{3+}$ becomes faster. The result of this process is the decrease in $\varepsilon^{\prime}$ with increasing frequency.

The variation of $\varepsilon^{\prime}$ and the calculated electric dipole moments $\mu^{\prime}($ Col.cm) as a function of sintering periods are listed in Tables (1) and (2) respectively. From the tables, it is clear that $\varepsilon^{\prime}$ increases with temperature for all sintering hours. Under constant temperature the smallest value of $\varepsilon^{\prime}$ is obtained at $20 \mathrm{~h}$. This means that, the density of packing may play a role in increasing and decreasing $\varepsilon^{\prime}$ with the sintering hours. This behavior is also assured from Table (2) which indicates the correlation between the electric dipole moment and the sintering periods.

Table (1). Sintering hours and $\varepsilon^{\prime}$ as a function of temperature and frequencies $(100 \mathrm{kHz}$ and $900 \mathrm{kHz})$ at $\mathrm{x}=0.1$.

\begin{tabular}{|lcllccl|}
\hline \multirow{2}{*}{$\boldsymbol{K}$} & \multicolumn{2}{c}{$\boldsymbol{\varepsilon}(\mathbf{8 h})$} & \multicolumn{2}{c}{$\boldsymbol{\varepsilon}^{\prime}(\mathbf{2 0 h})$} & \multicolumn{2}{c|}{$\boldsymbol{\varepsilon}^{\prime}(\mathbf{3 3 h})$} \\
\cline { 2 - 7 } & $\mathbf{1 0 0 k H z}$ & $\mathbf{9 0 0 k H z}$ & $\mathbf{1 0 0 k H z}$ & $\mathbf{9 0 0 k H z}$ & $\mathbf{1 0 0 k H z}$ & $\mathbf{9 0 0 k H z}$ \\
\hline $\mathbf{3 5 2}$ & 101.09 & 88.92 & 30.5 & 31.16 & 74.12 & 66.16 \\
$\mathbf{3 7 3}$ & 106.84 & 89.93 & 31.53 & 31.34 & 77.93 & 67.20 \\
$\mathbf{4 0 0}$ & 120.70 & 92.30 & 33.41 & 31.96 & 88.15 & 65.81 \\
$\mathbf{4 5 3}$ & 142.34 & 95.10 & 37.67 & 34.35 & 101.83 & 70.31 \\
$\mathbf{5 0 0}$ & 174.79 & 96.00 & 43.58 & 37.67 & 117.77 & 77.24 \\
$\mathbf{5 5 0}$ & 224.33 & 123.41 & 45.59 & 40.48 & 550.75 & 138.38 \\
$\mathbf{5 9 6}$ & 266.10 & 137.94 & 51.94 & 41.22 & 159.68 & 91.44 \\
\hline
\end{tabular}

Table (2). The electric dipole moment $\mu^{\prime}$ with the sintering hours as a function of temperature and frequencies $100 \mathrm{kHz}$ and $900 \mathrm{kHz}$.

\begin{tabular}{|ccccccc|}
\hline \multirow{2}{*}{$\boldsymbol{T} \boldsymbol{K}$} & \multicolumn{3}{c}{$\boldsymbol{\mu}^{\prime}($ Coul.cm)at300kHz} & \multicolumn{2}{c|}{$\mu^{\prime}$ (Coul.cm)at900kHz } \\
\cline { 2 - 7 } & $\boldsymbol{8} \boldsymbol{h}$ & $\mathbf{2 0 h}$ & $\mathbf{3 3 h}$ & $\mathbf{8 h}$ & $\mathbf{2 0 h}$ & $\mathbf{3 3 h}$ \\
\hline $\mathbf{3 5 2}$ & $7.8 \times 10^{-19}$ & $1.35 \times 10^{-18}$ & $1.14 \times 10^{-18}$ & $8.12 \times 10^{-19}$ & $1.35 \times 10^{-18}$ & $0.97 \times 10^{-18}$ \\
$\mathbf{4 0 0}$ & $7.94 \times 10^{-19}$ & $1.42 \times 10^{-18}$ & $1.14 \times 10^{-18}$ & $8.50 \times 10^{-19}$ & $1.43 \times 10^{-18}$ & $1.04 \times 10^{-18}$ \\
$\mathbf{5 2 0}$ & $7.71 \times 10^{-19}$ & $1.45 \times 10^{-18}$ & $0.94 \times 10^{-18}$ & $8.78 \times 10^{-19}$ & $1.50 \times 10^{-18}$ & $1.05 \times 10^{-18}$ \\
$\mathbf{5 6 0}$ & $7.47 \times 10^{-19}$ & $1.48 \times 10^{-18}$ & $0.90 \times 10^{-18}$ & $8.62 \times 10^{-19}$ & $1.53 \times 10^{-18}$ & $1.04 \times 10^{-18}$ \\
\hline
\end{tabular}


The most peculiar behavior in the hollandite structure is doubtless the short $\mathrm{Ba}-\mathrm{Ba}$ distance of $2.86 \mathrm{~A}^{\mathrm{o}}$ i.e. only a little more than twice the ionic radius of the $\mathrm{Ba}^{2+}$ ion [6]. Because of this peculiarity this distance may be varied with the variation of sintering hours resulting in a change in $\varepsilon^{\prime}$ and the electric dipole moment $\mu^{\prime}$.

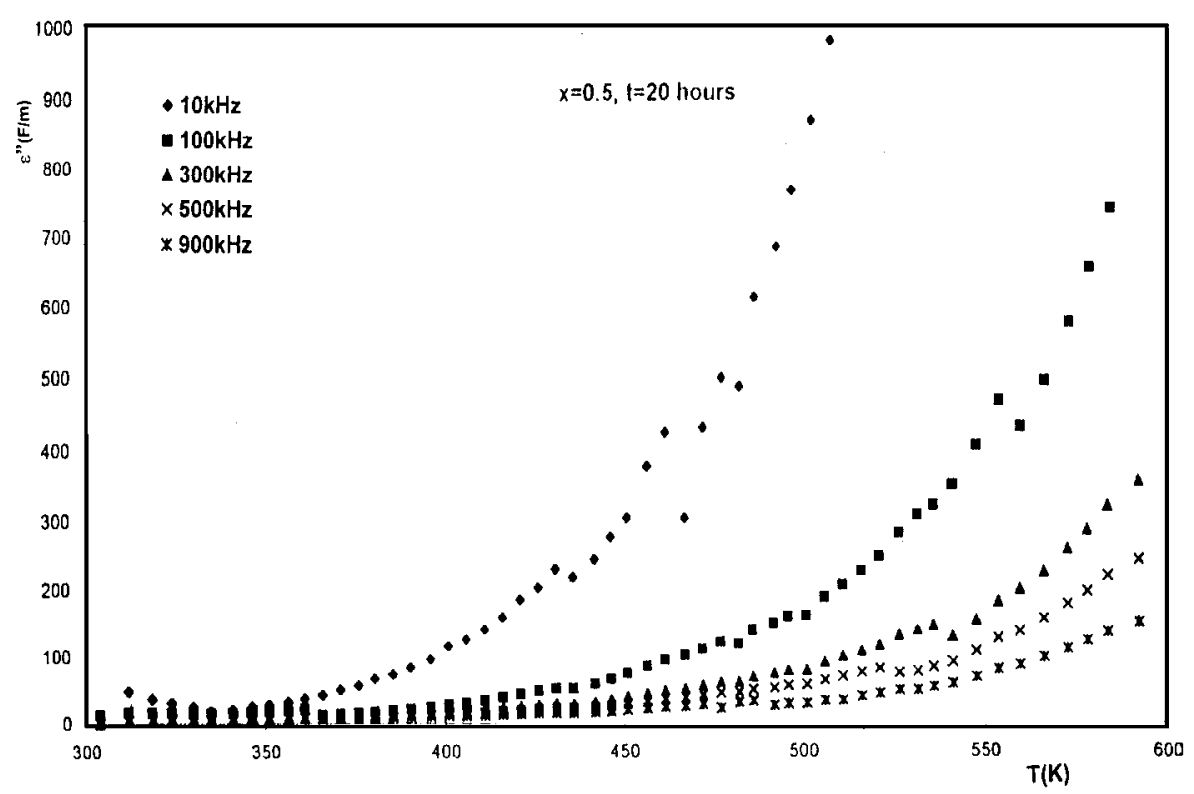

Fig. (2): The imaginary part for concentration $x=0.5$ and sintering time $t=20$ hour as a function dependence of the imaginary part of the dielectric constant $\varepsilon^{\prime \prime}$ on the absolute temperature of frequency.

The dependence of the imaginary part of the dielectric constant $\varepsilon^{\prime \prime}$ for $\mathrm{x}=0.5$ on absolute temperature at different frequencies is shown in Fig.(2). From the figure, it is clear that $\varepsilon^{\prime \prime}$ is almost constant up to $\cong 425 \mathrm{~K}$ after which it increases with increasing temperature. This is because, the thermal energy given to the system increases the motion of the dipoles and the energy dissipation due to their friction will be increased with the result of increasing $\varepsilon^{\prime \prime}$. Also $\varepsilon^{\prime \prime}$ decreases with increasing frequency, due to fast rotation of the dipoles under the action of increasing the field variation. Though, the dielectric loss increases and the energy required to overcome the resistance of the viscous medium will be reduced when the dipoles rotate through a unit angle [8]. 
Figure (3) is a typical curve that correlates the logarithm of ac. resistivity $(\ln \rho)$ and the absolute temperature as a function of frequency for $\mathrm{Ba}_{\mathrm{x}} \mathrm{Ti}_{4}$ ${ }_{2 x} \mathrm{Fe}_{2 \mathrm{x}} \mathrm{O}_{8}$,

$\mathrm{x}=0.5$ and at sintering time of 20 hours. A slight change in the slope is markedly observed at all frequencies. The calculated values of activation energy at low $\left(\mathrm{E}_{\mathrm{I}}\right)$ and high $\left(\mathrm{E}_{\mathrm{II}}\right)$ temperature regions are illustrated in the inset of Fig.(3). The inset shows that the activation energy decreases with increasing frequency, as a result of liberating more charge carriers from the trapping centers.

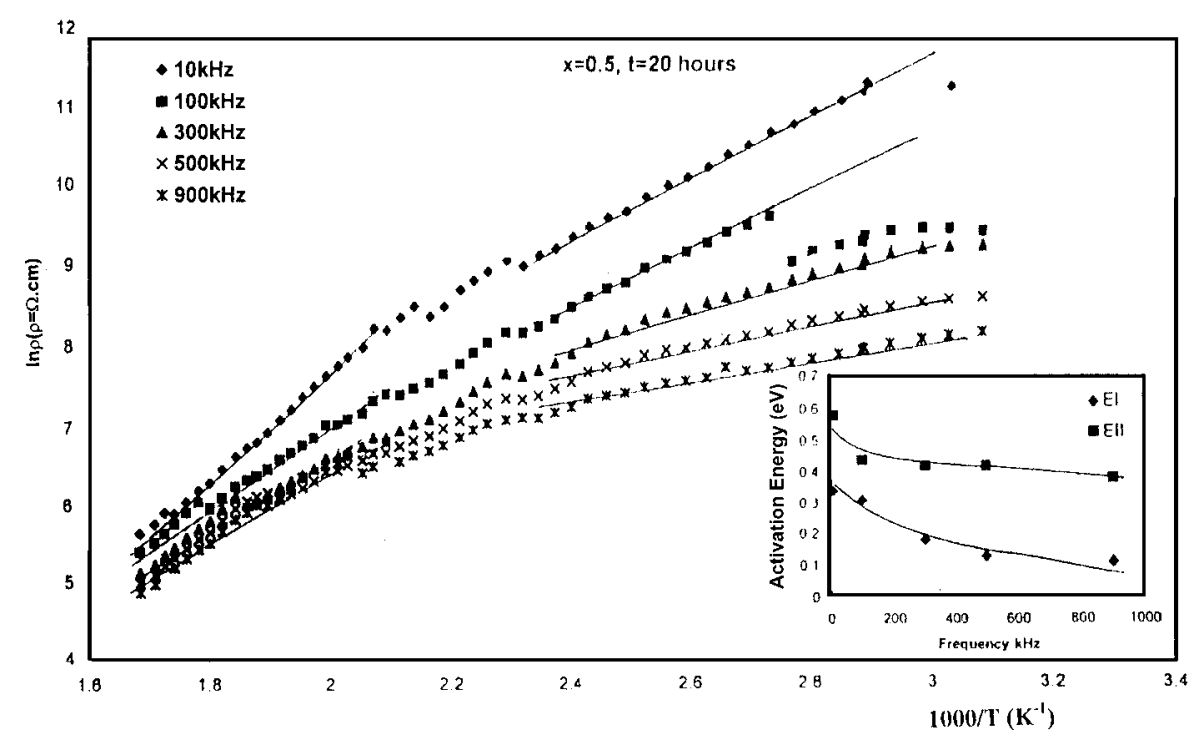

Fig. (3) : The relation between the logarithm of ac resistivity and the reciprocal of the absolute temperature for $\mathrm{x}=0.5$ and sintering time $\mathrm{t}=20 \mathrm{~h}$ as a function of frequency.

The inset:The variation of activation energy with frequency in the low $\left(E_{1}\right)$ and high $\left(E_{11}\right)$ temperature regions at $\mathrm{x}=0.5$ and $\mathrm{t}=20 \mathrm{~h}$.

The variation of the charge carriers mobility $(\mu)$ with temperature at different sintering periods is shown in Fig.(4), as calculated from the relation $\sigma=$ ne $\mu$ where "e" is the electronic charge and " $\mathrm{n}$ " is the charge carriers concentration $\left(n=2 \mathrm{~N}\right.$ where $\mathrm{N}$ is the number of dipoles; $\mathrm{N}=6.023 \times 10^{23}$ $\mathrm{xdensity} /$ molecular weight). The values of the charge carrier mobility at $\mathrm{x}=0.3$ and frequency of $900 \mathrm{kHz}$ for different sintering hours increases with increasing temperature. This behavior is similar to that exhibited by many semiconductors [9]. Also, it can be seen that the charge carrier mobility change with the sintering periods and the product $\left\{\varepsilon^{\prime} \rho^{1 / 2}\right\}[10]$ remains nearly constant. 


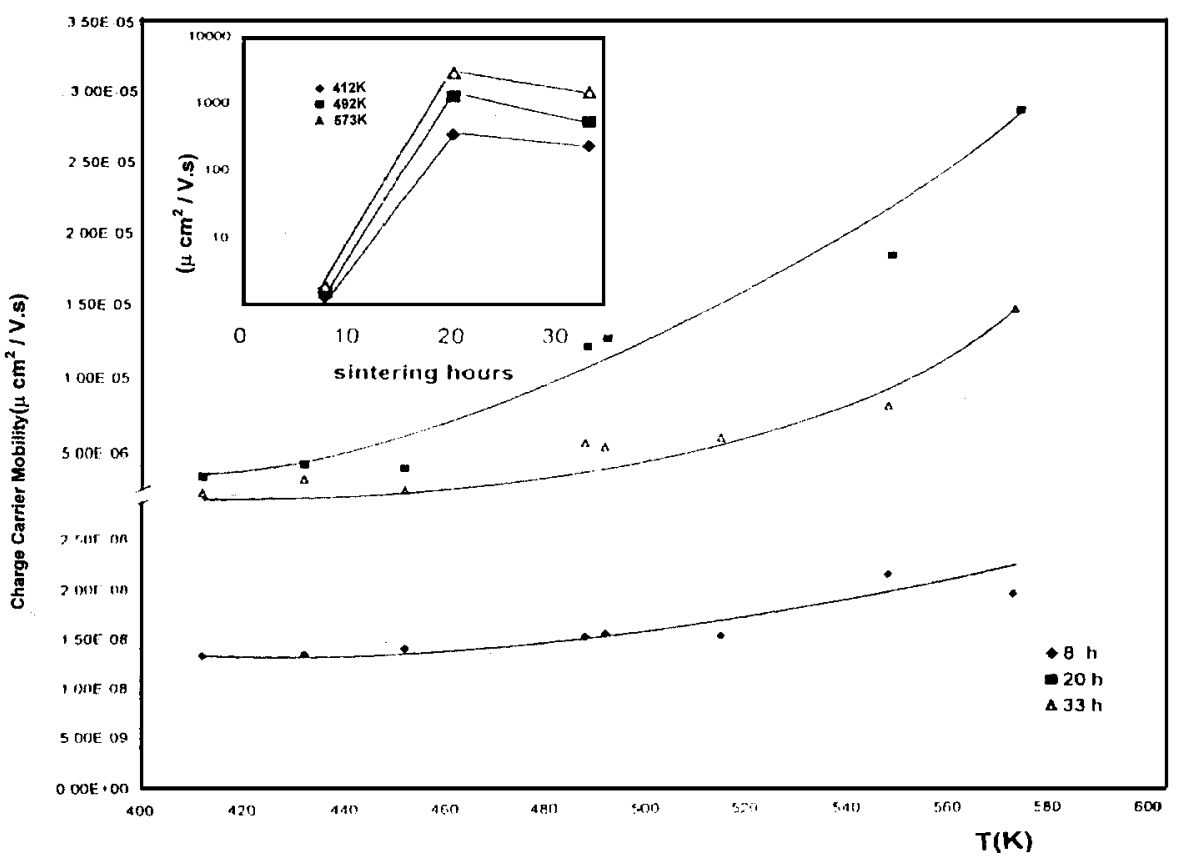

Fig. (4) : The relation of the charge carrier mobility with the absolute temperature as a function of sintering time $(8 \mathrm{~h}, 20 \mathrm{~h}$ and $33 \mathrm{~h})$.

The inset: A longitudinal view of the charge carrier mobility with sintering time at three different temperatures.

In order to obtain information about the type of charge carriers in the investigated samples, the Seebeck coefficient studies were carried out. Fig. (5) shows a plot of the Seebeck coefficient versus temperature for different sintering times and different concentrations. For $x=0.1$ and sintering time of $8 \mathrm{~h}$, $\mathrm{Ba}^{2+}$ ions need a small energy (ionization potential of $\mathrm{Ba}=5.212 \mathrm{~V}$ while that for $\mathrm{Fe}$ is $7.870 \mathrm{~V}$ ) to participate in the conduction process. At higher temperature region, the electrons at lower depths (these electrons are trapped in vacant sites at different depths) begin to participate in the conduction mechanism nearly at $\mathrm{T}=377 \mathrm{~K}$. When the sintering time is increased to $20 \mathrm{~h}$ the Seebeck coefficient behavior remains the same. Increasing the sintering time to $33 \mathrm{~h}$ renders the Seebeck coefficient nearly constant with a negative sign, which may be due to the valence exchange between $\mathrm{Fe}^{3+}$ and $\mathrm{Fe}^{2+}$. Generally, it was found that by increasing the concentration from 0.1 to 0.3 , no detectable variation in the type of conduction appears. In the case of $x=0.5$ the Seebeck coefficient appears with a negative sign and the electron exchange would take place between $\mathrm{Ti}^{4+}$ and $\mathrm{Ti}^{2+}$ which plays a role in the conduction mechanism. 


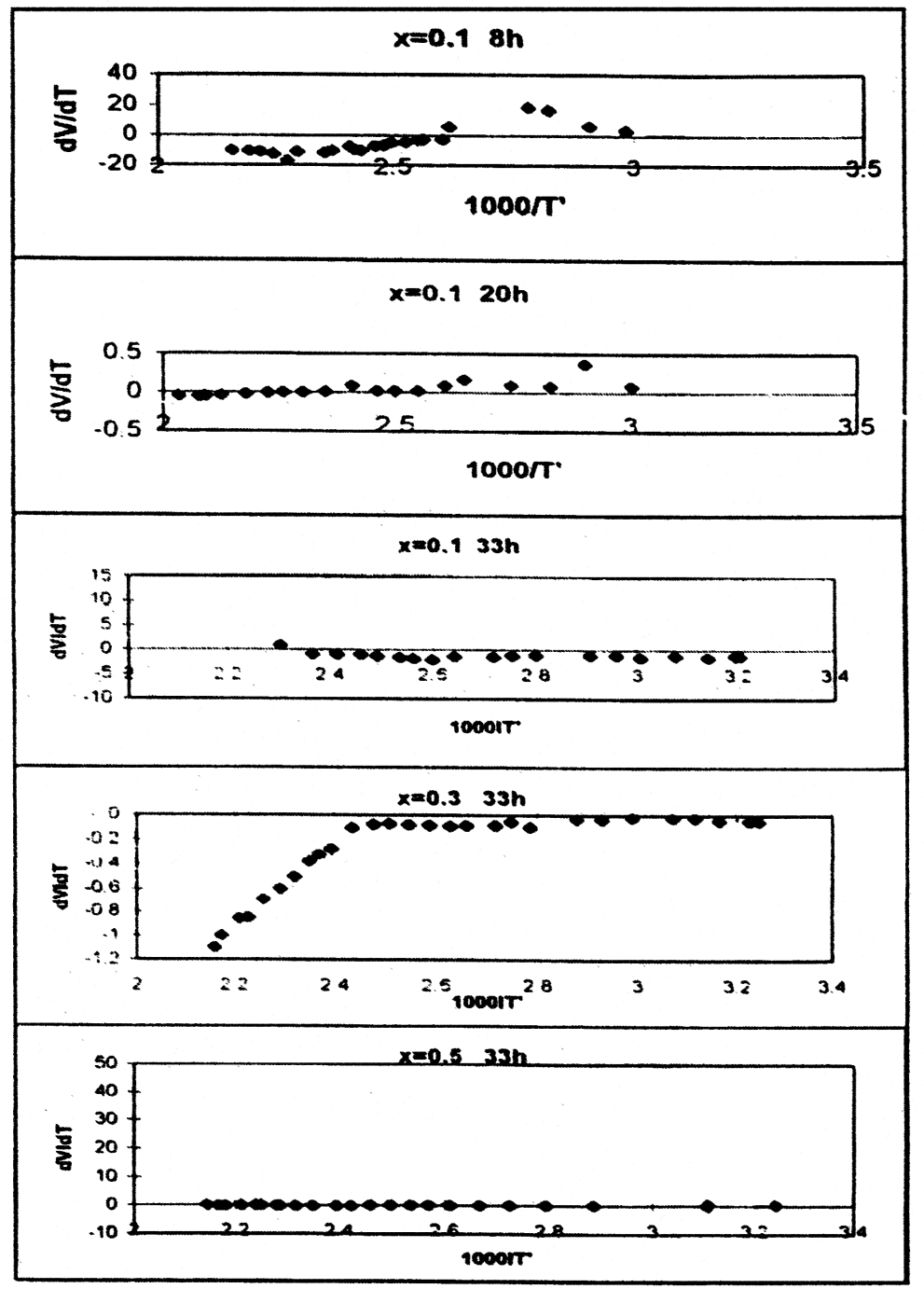

Fig. (5): The relation between Seebeck coefficient and the reciprocal of the average absolute temperature at different sintering hours and concentrations $(\mathrm{t}=8 \mathrm{~h}, 20 \mathrm{~h}, 33 \mathrm{~h}$ and $\mathrm{x}=0.1,0.3,0.5)$.

\section{References}

1. M. C. Cadee and G. C. Uerschoor, Acta. Cryst., B 34, 3554 (1978).

2. M. C. Cadee and A. Prodan, Mat. Res. Bull., 14, 613 (1979).

3. Y.Abbas and M.A.Ahmed, Egypt.J.Sol.4, 17 (1982).

4. H. B.G.Casimir ,J.Phys.Rad,8,(1959) 
5. J.Smit and H.P.J.Wijn, ferrite, JohnWiley and Sons (1959).

6. A.Byström and A.M.Byström, Acta.Cryst.,3, 146 (1950).

7. 7.M.A.Ahmed, K.A.Darwish, H.Mikhail, M.Mounir and E.H.El-Khawas, Physica Scripta, 55, 750 (1997).

8. K. Latha, K.Satya Mohan, and D. Ravinder., phys. stat. sol. (a) 142, K 103 (1994).

9. Van Uitert,L.G., J.Chem.Phys., 24, 306 (1956).

10. D.Ravinder, Phys. Stat. Sol. (a) 129, 549(1992). 